\title{
Análise pós-operatória tardia da artrodese por via posterior em pacientes ASIA/Frankel e com trauma raquimedular toracolombar em serviço universitário
}

\author{
Tiago de Paiva Cavalcante ${ }^{1}$, Siegfried Pimenta Kuehnitzsch ${ }^{1}$, Emerson Oliveira Barbosa ${ }^{2}$, \\ Otacilio Moreira Guimarães ${ }^{3}$, Gustavo Veloso Lages ${ }^{4}$, George Santos dos Passos ${ }^{5}$
}

Hospital Universitário Alzira Velano - Alfenas, MG, Brasil.

\section{RESUMO}

Objetivo: O propósito deste estudo foi avaliar retrospectivamente o tratamento cirúrgico de pacientes vitimas de trauma raquimedular desse segmento sem déficit neurológico, quanto à evolução da dor local e à deformidade cifótica local e regional do segmento acometido, em um serviço universitário. Método: Foram utilizados dados de prontuário e avaliações clínicas e radiológicas de 16 pacientes submetidos à artrodese pedicular por via posterior, no período de maio de 2003 a dezembro de 2006, operados há pelo menos dois anos. Resultados: A média de idade foi de 43,43 $\pm 11,44$ anos e o nível mais acometido L1. O mecanismo principal do trauma foi queda de altura. Após realização de raios $X$ em perfil e cálculo dos ângulos local sagital (ALS) e regional sagital (ARS), de pós-operatório precoce e tardio, não houve diferença estatisticamente significativa entre os dados obtidos, apesar da diminuição de $-15,3^{\circ}$ para $-10,7^{\circ}$ nas cifoses locais e do aumento de $-13,7^{\circ}$ para $15,9^{\circ}$ nas cifoses regionais. Houve diferença estatística na avaliação de dor pela Escala Analógica de Dor (EAD), entre o pré-operatório e o pós-operatório tardio (maior que dois anos), com redução de quatro vezes da intensidade da dor entre os pacientes analisados $(p<0,05)$. Conclusão: $A$ artrodese pela via posterior é uma proposta alternativa de tratamento quando se discute a dor desses pacientes. Fica a necessidade de abranger um número maior de pacientes com essa patologia, a fim de dispor dados mais fidedignos, respaldando o tratamento como uma alternativa viável no manejo de pacientes com fraturas toracolombares e neurologicamente intactos.

\section{PALAVRAS-CHAVE}

Traumatismos da coluna vertebral, artrodese, traumatismos da medula espinal/cirurgia.

\begin{abstract}
Postoperative late analysis of posterior arthrodesis in patients ASIA/Frankel e with thoracolumbar spine trauma in university hospital

Objective: The aim of this study was a retrospective valuation of surgical treatment of patients with spinal thoracolumbar spine without neurological deficit, for that local pain, local and regional kyphotic deformity at an university hospital. Method: There were used hospital datum from 16 operated patients and clinical and radiological evaluation submitted a pedicular screw instrumentation from May 2003 until December 2006, operated for at least 2 years. Results: The mean age was $43.43 \pm 11.44$ years and the most fractured level $L 1$. The principal mechanism was height fall. After the $x$-rays realized and calculated the local sagittal angle and regional sagittal angle, there's no significative difference between them, despite the reduction of $-15.3^{\circ}$ to $-10.7^{\circ}$, at local kyphosis and augmentation of $-13.7^{\circ}$ to $15.9^{\circ}$ at regional kyphosis. There was statistics difference at pain evaluation using the Analogic Pain Scale, between the pre-operative and postoperative (beyond two years), with four times reduction of intensity pain among the analyzed patients ( $p$ < 0.05). Conclusion: The arthrodesis by posterior approach is an alternative proposal of treatment when discussing the pain of these patients. It is the need to include a larger number of patients with this pathology, in order to dispose more data reliable, endorsing the treatment as a viable alternative in the management of patients with thoracolumbar fractures and neurologically intact.
\end{abstract}

\section{KEYWORDS}

Spinal injuries, arthrodesis, spinal cord injuries/surgery.

1 Neurocirurgião da Fundação de Neurologia e Neurocirurgia - Instituto do Cérebro, Salvador, BA; ex-residente do Hospital Universitário Alzira Velano (HUAV), Alfenas, MG, Brasil.

2 Neurocirurgião e ex-preceptor da residência de neurocirurgia do HUAV, Alfenas, MG, Brasil.

3 Neurocirurgião chefe da residência de neurocirurgia do HUAV, Alfenas, MG, Brasil.

4 Residente de neurocirurgia do HUAV, Alfenas, MG, Brasil.

5 Acadêmico de medicina da Escola Bahiana de Medicina e Saúde Pública, Bahia, Brasil. 


\section{Introdução}

Os traumas na região da coluna vertebral compreendem uma parcela significativa da população, além de promover altos custos à sociedade. Estima-se que nos Estados Unidos existam 150.000 pessoas incapacitadas vítimas de algum trauma vertebral e que 10.000 novos casos se adicionem a esse grupo por ano. ${ }^{1,2}$ Ao se considerar a coluna toracolombar, o segmento mais acometido é o da junção toracolombar (T11-L1), seguido pelos níveis torácicos (T1-T10) e lombares (L2-L5). ${ }^{3}$ Passagem de uma área de menor para de lares a partir de T11, permitindo maior movimento nesse plano e aumentando a resistência rotacional em relação à coluna torácica com suas facetas articulares num plano coronal, são fatores da maior incidência de fraturas nessa região. ${ }^{4,5}$ Adicione-se a isso a menor força que os ligamentos longitudinais posteriores e amarelo, e as facetas articulares em relação à coluna lombar. As lesões medulares nesse segmento podem afetar a medula espinhal baixa, cone medular e cauda equina, com uma variedade de lesões de prognósticos diversos.

Seguindo os critérios de estabilidade/instabilidade da AOSpine (Figura 1), as fraturas do tipo A são derivadas de uma força de compressão axial, associada ou não à flexão, mantendo intactos os ligamentos posteriores, portanto não havendo movimentação no plano sagital. As fraturas do tipo B e seus subtipos envolveriam um mecanismo de flexão-distração com rotura e alongamento dos elementos posteriores, ou hiperextensão com ou sem cisalhamento anterior. No tipo Chá uma rotação e associação com os outros tipos de fratura, A ou B. ${ }^{6}$

Apesar de as fraturas do tipo A pela classificação $\mathrm{AO}$ serem as mais frequentes $(66 \%)^{6}$, continuam sendo as que mais despertam opiniões divergentes quanto ao seu manejo. Não há dúvida de que, em pacientes com deterioração neurológica progressiva, a descompressão neurocirúrgica está indicada. ${ }^{7}$ Fraturas com mais de $50 \%$ de colapso do corpo anterior da vértebra, ou com mais de $20^{\circ}$ de angulação sagital ou mais de $40 \%-50 \%$ de comprometimento do canal vertebral no nível da fratura em pacientes neurologicamente intactos, são todos critérios para tratamento cirúrgico, uma maneira indireta de indicar a perda da tensão da coluna posterior e, portanto, da estabilidade sagital da coluna toracolombar. ${ }^{1,3,-10}$

As instrumentações pediculares curtas são as mais utilizadas e difundidas para abordagem cirúrgica posterior das fraturas toracolombares ao redor do mundo. Realizadas com um nível acima e outro abaixo, com colocação de um parafuso através do pedículo das vértebras íntegras, procuram restabelecer o balanço sagital e estabilizar a fratura, limitando o número de segmentos instrumentados ao mínimo necessário, uma vez que não há diferenças entre construções com dois níveis acima ou abaixo destas. ${ }^{7}$

A importância do estudo desse assunto está no fato de que ainda não há concordância total quanto ao tratamento ideal desses pacientes, principalmente no pós-operatório tardio.
Força de compressão

Fraturas por compressão e explosão

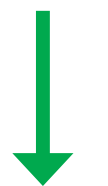

Tipo A - Compressão A.1 - Fraturas impactadas

A.1.1 - Impactação da placa terminal

A.1.2 - Encunhamento

A.1.3 - Colapso do corpo vertebral

A.2 - Split (separação)

A.2.1 - Sagital

A.2.2 - Coronal

A.2.3 - Pinça

A.3 - Explosão

A.3.1 - Incompleta

A.3.2 - Explosão-separação

A.3.3 - Completa
Força de tensão

Lesões com traço de

ruptura transverso

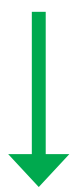

Tipo B - Lesão por distração

B.1 - Lesão posterior ligamentar

B.1.1 - Com rotura transversa do disco

B.1.2 - Associada à fratura do tipo $\mathrm{A}$

B. 2 - Lesão posterior óssea

B.2.1 - Fratura transversa da vértebra (Chance)

B.2.2 - Espondilólise com lesão do disco

B.2.3 - Espondilólise com fratura do tipo A

B.3 - Lesão anterior - Hiperextensão

B.3.1 - Hiperextensão-subluxação

B.3.2 - Hiperextensão-espondilólise

B.3.3 - Luxação posterior

\section{Torque axial}

Lesões rotacionais

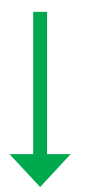

Tipo C - Rotação

C.1 - Lesão tipo A + rotação

C.1.1 - Impactada

C.1.2 - Separação

C.1.3 - Explosão

C.2 - Lesão tipo B + rotação

C.2.1 - Lesão B.1 + rotação

C.2.2 - Lesão B. 2 + rotação

C.2.3 - Lesão B.3 + rotação

C. 3 - Cisalhamento-rotação

C.3.1 - Fratura do tipo slice

C.3.2 - Fratura oblíqua

Figura 1 - Classificação das fraturas toracolombares adotada pela AOSpine, segundo os critérios de Magerl (com permissão). 


\section{Materiais e métodos}

Estudo retrospectivo de 16 pacientes vítimas de traumatismo raquimedular no segmento de T10-L2, sem déficits neurológicos, submetidos à artrodese cirúrgica por via posterior, no período entre maio de 2003 e dezembro de 2006, operados há pelo menos dois anos. Os pacientes foram incluídos seguindo os seguintes critérios, baseados em Siebenga et al. ${ }^{11}$ : fratura traumática de T10-L2, tipo A pela AO (compressão), com acunhamento anterior maior que $50 \%$ e/ou cifose maior que $20^{\circ}$ e/ou estenose de canal vertebral maior que $40 \%$, sem déficit neurológico (ASIA/Frankel E), período de pós-operatório não inferior a dois anos.

Os critérios de exclusão foram fratura tipo A.1.1 pela $\mathrm{AO}$, gestação, fratura patológica ou osteoporótica, pacientes com doença terminal, pacientes com história de cirurgia prévia na coluna, pacientes com histórico psiquiátrico recente, pacientes em uso de drogas ou outras substâncias ilegais, ou pacientes apresentando injúria que pudesse interferir com o tratamento proposto. O trabalho foi submetido à comissão de ética do hospital e todos os pacientes foram informados e consentiram.

Os pacientes foram submetidos a um questionário próprio, que avaliava dados demográficos como idade, mecanismo da lesão, segmentos lesionados e fixados cirurgicamente e dados sobre evolução da dor no local fraturado no período e aplicados na Escala Analógica Visual de Dor. Continha, ainda, dados coletados em exames de imagem do pós-operatório imediato (menor que três meses) e exames de imagem do pós-operatório tardio (maior que dois anos), relacionando informações dos ângulos locais e regionais nos segmentos afetados.

\section{Avaliação clínica e método cirúrgico}

Após a admissão e o estudo radiológico completo com radiografias de coluna toracolombar em incidências anteroposterior e perfil e tomografia de coluna helicoidal (Figura 2), os pacientes vítimas de traumatismo raquimedular com fratura no segmento T10L2 e indicação neurocirúrgica, sem déficit neurológico, foram submetidos à abordagem cirúrgica precoce. Seguindo a técnica descrita e consagrada na literatura, foi realizada laminectomia do nível/níveis fraturados e fixados parafusos pediculares um ou dois níveis acima ou abaixo e conectados a barras de fixação longitudinais e, sempre que possível, inserida barra de fixação transversal. Foi realizado enxerto autólogo ósseo retirado das laminectomias sobre o trajeto das barras longitudinais, especialmente nos pontos de inserção do parafuso pedicular. A alta hospitalar ocorreu em 3-4 dias, sem dor e após raios $\mathrm{X}$ de controle, para acompanhamento ambulatorial especializado. Foi prescrita fisioterapia motora para todos os pacientes, e estes não utilizaram colete de Jewite no pós-operatório.

Realizou-se acompanhamento ambulatorial de neurocirurgia, em pelo menos dois anos de pós-operatório. $\mathrm{Na}$ Escala Analógica de Dor, o valor 0 foi dado para dor inexistente e 10 para a maior dor possível. Solicitados raios $\mathrm{X}$ de coluna toracolombar em perfil neutro e dinâmico em flexão e extensão (Figuras 3 e 4). Os exames em flexão/ extensão foram realizados com o paciente em ortostatismo

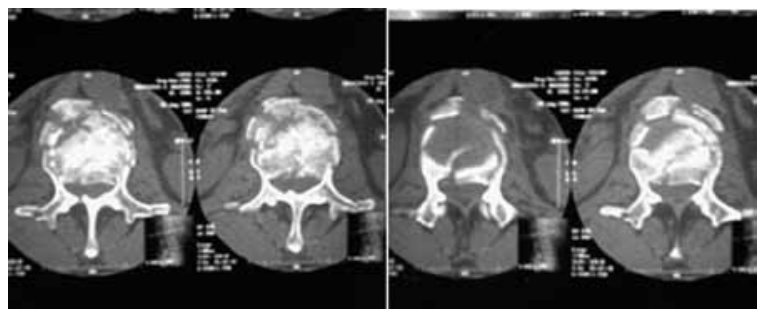

Figura 2 - Imagem de tomografia computadorizada de paciente vítima de trauma raquimedular (com autorização).

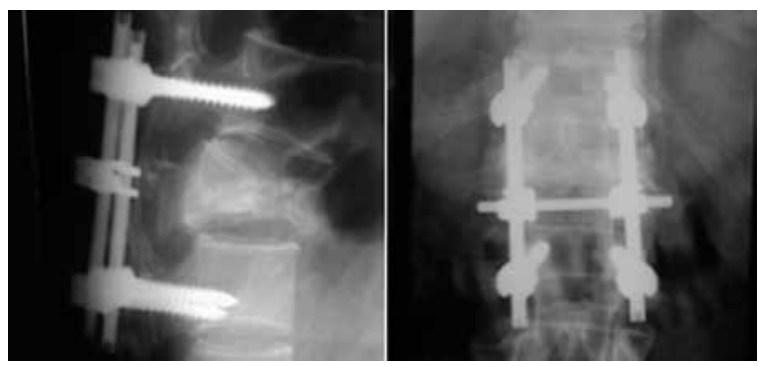

Figura 3 - Imagem de raios X de PO imediato de artrodese por via posterior de T12-L2 (com autorização).



Figura 4 - Imagem de raios $X$ de $P O$ tardio de artrodese por via posterior de T12-L2 do paciente acima (com autorização). 
e flexão sagital do tronco forçada e hiperextensão forçada. A angulação cifótica foi indicada com um sinal de menos. Ângulos sagitais locais e regionais foram mensurados nos raios $\mathrm{X}$ em perfil. O ângulo local é a medida do ângulo entre os platôs superior e inferior da vértebra fraturada. A angulação sagital regional é dada pelo ângulo entre o platô superior da vértebra superior à vértebra fraturada e o platô inferior, da vértebra inferior à fraturada. Todos os ângulos foram medidos em posição neutra, em flexão e extensão.

Para avaliar a possível relação entre o grau de cifose precoce e tardia após a artrodese por via posterior e a relação do trauma e cifose com o grau de dor dos pacientes, foi utilizado o teste do Qui-quadrado.

\section{Resultados}

Foi realizada análise de dados de todos os 16 pacientes coletados (Tabela 1): a idade média foi de 43,43 $\pm 11,44$ anos, com idades variando dos 20 aos 62 anos; todos os pacientes foram classificados pela $\mathrm{AO}$ como tipo A; o nível mais acometido foi L1 (68,75\%), isoladamente ou associado com outras fraturas, enquanto o mecanismo de trauma mais comum foi queda de altura, em $62,3 \%$ dos casos. No entanto, não houve relação estatisticamente significativa entre o tipo de fratura pela AO e o mecanismo do trauma.

Os resultados obtidos durante o acompanhamento dos pacientes mostraram que houve diminuição da média da cifose local de $-15,3^{\circ}$, no pós-operatório precoce (menos de dois meses após a cirurgia), para $-10,7^{\circ}$, no pós-operatório tardio (mais de dois anos após a cirurgia) $(\mathrm{p}>0,05)$. Em relação aos ângulos de cifose regionais, não houve diferença estatisticamente entre o pós-operatório precoce e o tardio $\left(-13,7^{\circ} \mathrm{x}\right.$ $-15,9^{\circ}$, respectivamente). Quando se analisaram os dados obtidos relacionando os raios $\mathrm{X}$ dinâmicos, em busca de instabilidades (diferença maior que $10^{\circ}$ entre as incidências obtidas), ou seja, pseudoartrose, houve aumento da cifose local em flexão, mas não estatisticamente significante $\left(-10,7^{\circ}\right.$ em posição neutra $\mathrm{x}-18,6^{\circ}$ em flexão), e praticamente não se alteraram nas imagens obtidas em extensão. Nos ângulos de cifose regionais, não houve alteração significativa, nas aquisições em repouso, flexão e extensão (Figura 5 e Tabela 2).

$\mathrm{Na}$ análise funcional pela Escala Analógica de Dor (EAD), houve redução estatisticamente significativa entre os valores dados para a dor pré e pós-operatória tardia, ou seja, os pacientes melhoraram pelo menos quatro vezes da dor no período ( $\mathrm{p}<0,05)$, sem haver relação com as alterações biomecânicas observadas nos exames de imagem realizados (Tabela 3 ).

\begin{tabular}{|c|c|c|c|c|c|c|}
\hline$N^{0}$ do caso & Sexo & Idade (anos) & Nível & Tipo AO & Causa & Segmentos artrodesados (a) \\
\hline PAC 1 & M & 39 & L1-L2 & A 1.2 & Queda de altura & T12-L1-L2-L3 \\
\hline PAC 2 & $\mathrm{~F}$ & 56 & L1 & A 3.1 & Queda da própria altura & T12-L1-L2 \\
\hline PAC 3 & M & 47 & L1 & A 3.1 & Queda de altura & T12-L1-L2 \\
\hline PAC 4 & M & 48 & L1 & A1.2 & Queda de altura & T12-L1-L2 \\
\hline PAC 5 & M & 50 & $\mathrm{~T} 12$ & A 1.3 & Queda de altura & T11-T12-L1 \\
\hline PAC 6 & $\mathrm{~F}$ & 32 & $\mathrm{~T} 12$ & A 1.2 & Acidente ciclístico & T11-T12-L1 \\
\hline PAC 7 & M & 28 & $\mathrm{~T} 12$ & A1.2 & Queda de altura & T11-T12-L1 \\
\hline PAC 8 & M & 47 & L1 & A 1.2 & Queda de altura & T11-T12-L1 \\
\hline PAC 9 & M & 48 & $\mathrm{~T} 12$ & A1.2 & Trauma direto & T11-T12-L1 \\
\hline PAC 10 & M & 53 & $\mathrm{~T} 12$ & A 1.2 & Acidente automobilístico & T11-T12-L1 \\
\hline PAC 11 & M & 38 & L1 & A 1.2 & Acidente automobilístico & T11-T12-L1 \\
\hline PAC 12 & M & 43 & L1 & A3.1 & Queda de altura & T12-L1-L2 \\
\hline PAC 13 & M & 54 & L1 & A 3.1 & Queda de altura & T12-L1-L2 \\
\hline PAC 14 & M & 20 & $\mathrm{~T} 12-\mathrm{L} 1$ & A 3.1 & Queda de altura & T11-T12-L1-L2 \\
\hline PAC 15 & M & 62 & L1-L2 & A 3.1 & Queda de altura & T12-L1-L2-L3 \\
\hline PAC 16 & M & 30 & $\mathrm{~T} 11-\mathrm{L} 1$ & A1.2 & Atropelamento & T10-T11-T12-L1-L2 \\
\hline
\end{tabular}

(a) Letras em negrito: segmentos fixados e com parafusos pediculares; letras em itálico: segmentos fixados mas sem parafusos pediculares. 


\begin{tabular}{|c|c|c|c|c|c|c|c|c|}
\hline Caso & ALS POI & ARS POI & ALS POT & ARS POT & $\begin{array}{l}\text { ALS NO POT } \\
\text { (flexão) }\end{array}$ & $\begin{array}{l}\text { ARS NO POT } \\
\quad \text { (flexão) }\end{array}$ & $\begin{array}{l}\text { ALS NO POT } \\
\text { (extensão) }\end{array}$ & $\begin{array}{l}\text { ARS NO POT } \\
\text { (extensão) }\end{array}$ \\
\hline 01 & $-09^{\circ}$ & $-06^{\circ}$ & $-16^{\circ}$ & $-12^{\circ}$ & $-13^{\circ}$ & $-10^{\circ}$ & $-13^{\circ}$ & $-07^{\circ}$ \\
\hline 02 & $-08^{\circ}$ & $-18^{\circ}$ & $-11^{\circ}$ & $-10^{\circ}$ & $-09^{\circ}$ & $-27^{\circ}$ & $-07^{\circ}$ & $-17^{\circ}$ \\
\hline 03 & $-19^{\circ}$ & $-15^{\circ}$ & $-15^{\circ}$ & $-23^{\circ}$ & $-18^{\circ}$ & $-23^{\circ}$ & $-21^{\circ}$ & $-07^{\circ}$ \\
\hline 04 & $-21^{\circ}$ & $-28^{\circ}$ & $-14^{\circ}$ & $-32^{\circ}$ & $-24^{\circ}$ & $-24^{\circ}$ & $-23^{\circ}$ & $-21^{\circ}$ \\
\hline 05 & $-22^{\circ}$ & $-29^{\circ}$ & $-20^{\circ}$ & $-28^{\circ}$ & $-09^{\circ}$ & $-34^{o}$ & $-12^{\circ}$ & $-32^{\circ}$ \\
\hline 06 & $-07^{\circ}$ & $-08^{\circ}$ & $-12^{\circ}$ & $-15^{\circ}$ & $-06^{\circ}$ & $-13^{\circ}$ & $-08^{\circ}$ & $-14^{\circ}$ \\
\hline 07 & $-14^{\circ}$ & $-05^{\circ}$ & $-14^{\circ}$ & $-05^{\circ}$ & $-13^{\circ}$ & $-13^{\circ}$ & $-10^{\circ}$ & $-12^{\circ}$ \\
\hline 08 & - & - & - & - & - & - & - & - \\
\hline 09 & $-17^{\circ}$ & $-10^{\circ}$ & - & - & - & - & - & - \\
\hline 10 & - & - & - & - & - & - & - & - \\
\hline 11 & $-20^{\circ}$ & $-11^{o}$ & - & - & - & - & - & - \\
\hline 12 & $-16^{\circ}$ & $-01^{\circ}$ & $-04^{\mathrm{o}}$ & $-02^{\circ}$ & $-08^{\circ}$ & $-05^{\circ}$ & $-09^{\circ}$ & $-02^{\circ}$ \\
\hline 13 & - & - & - & - & - & - & - & - \\
\hline 14 & - & - & - & - & - & - & - & - \\
\hline 15 & - & - & - & - & - & - & - & - \\
\hline 16 & - & - & - & - & - & - & - & - \\
\hline
\end{tabular}

ALS: ângulo local sagital; ARS: ângulo regional sagital; POI: pós-operatório imediato; POT: pós-operatório tardio; PRE-OP: pré-operatório.

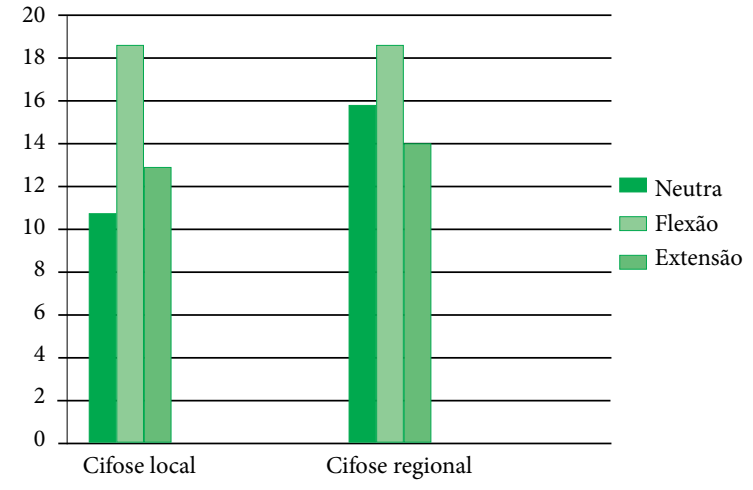

Eixo numérico com valores negativos.

Figura 5 - Relação entre os ângulos de deformidade tardia.

\section{Discussão}

Apenas um terço das fraturas toracolombares por trauma é de tratamento cirúrgico. Dessas, a maioria é do tipo A pela $\mathrm{AO}$, mas nem por isso existe consenso entre as diversas abordagens. Siebenga et al. ${ }^{11}$ concluíram, a partir de um estudo prospectivo, que apenas as fraturas do tipo A3 se beneficiariam de abordagem cirúrgica, enquanto nos tipos A1 e A2 não puderam concluir se um tratamento seria melhor que o outro.

Nenhum paciente com fratura tipo A1.1 foi submetido a tratamento cirúrgico. No entanto, os autores não têm a intenção de discutir as indicações de tratamento conservador ou cirúrgico. Os critérios descritos na literatura vigente $\mathrm{e}^{1,3,7-10} \mathrm{e}$ aceitos na prática neurocirúrgica

\begin{tabular}{lcc}
\hline & \multicolumn{3}{c}{ Tabela 3-Valores da Escala Analógica de Dor } \\
\hline Caso & EAD PRE-OP & EAD POT \\
\hline 01 & 06 & 00 \\
02 & 10 & 03 \\
03 & 06 & 01 \\
04 & 04 & 00 \\
05 & 05 & 02 \\
06 & 05 & 02 \\
07 & 08 & 04 \\
08 & - & - \\
09 & - & - \\
10 & - & - \\
11 & - & - \\
12 & 08 & 02 \\
13 & - & - \\
14 & - & - \\
15 & 09 & 01 \\
16 & 07 & 01 \\
\hline
\end{tabular}

PRE-OP: pré-operatório; POT: pós-operatório tardio.

foram os utilizados para o tratamento desses pacientes. Os autores acreditam que um tratamento que possibilite o retorno mais precoce às atividades de vida do paciente, quando não contraindicado, certamente tem mais benefícios, além do que uma técnica que permita menor morbidade pós-operatória (em contrapartida de uma abordagem cirúrgica pela via anterior), associada a acompanhamento médico e fisioterápico específico, respalde ainda mais a via posterior como tratamento ideal para esse grupo de pacientes. 
Além da decisão de abordar ou não pacientes sem déficits cirurgicamente, a via cirúrgica ideal ainda não está determinada totalmente. Vaccaro et al. ${ }^{12}$, após um estudo realizado com 20 centros de referência no trauma em sete países, separaram esses pacientes sem déficit em dois grupos: com evidência radiológica de ruptura dos ligamentos posteriores e sem evidência radiológica de lesão. Naqueles, uma situação vista mais comumente em fraturas do tipo A pela $\mathrm{AO}$, com compressão ou explosão, a via preferida foi com laminectomia e artrodese por via posterior, enquanto nestes, sem evidência de lesão, $59 \%$ optaram por indicar abordagem pela via posterior, principalmente se a decisão cirúrgica foi realizada com o intuito de acelerar a reabilitação do paciente, visto que as abordagens pela via anterior da coluna toracolombar são mais invasivas e mais complexas de serem realizadas, além do pós-operatório mais prolongado desses pacientes.

Em nosso Serviço, os indivíduos mais propensos são, na maioria, os homens, dado confirmado na casuística do autor, com $87,5 \%$ dos acidentes envolvendo o sexo masculino. Interessante notar que, apesar do número limitado de pacientes incluídos neste trabalho, os acidentes automobilísticos, traumas diretos (agressão física) e quedas de altura foram todos mecanismos envolvendo homens. A idade média de 43,5 anos foi semelhante à literatura para o segmento toracolombar, em torno dos 40 anos, de acordo com Bracken et al. ${ }^{13}$, Masini ${ }^{14}$, Souza et al. ${ }^{15}$, Zaninelli et al. ${ }^{16}$ e Campos et al. ${ }^{17}$, mas superior ao da média total para os traumatismos da coluna vertebral, de 35 anos. O segmento mais acometido em nosso Serviço, nesse grupo específico de pacientes, foi L1, diferente da frequência geral, que aponta os níveis T12 e L2 como os mais comprometidos. ${ }^{18}$

Com relação ao pequeno número de pacientes, vale destacar que a grande maioria dos pacientes atendidos em nosso Serviço vítimas de traumatismo raquimedular no segmento toracolombar é admitida com déficit, especialmente em ASIA/Frankel A. Com relação ao tempo utilizado para iniciar a avaliação pós-operatória, valeu-se do período mínimo como aceito para artrodese e consolidação da fratura, que foi de três meses. Esse tempo pode variar de 3 a 12 meses de acordo com protocolos próprios.

Ao analisar a deformidade regional a partir das angulações calculadas, em um paciente houve piora significativa da cifose. Após se analisarem os exames de imagem do período de avaliação, ficou claro um achatamento significativo da vértebra imediatamente superior ao segmento artrodesado, exatamente T11. A eliminação da mobilidade dos segmentos artrodesados pode levar a uma carga concentrada nos níveis adjacentes. Tal fato tem sido discutido por diversos autores. Enquanto alguns acreditam que há aumento da mineralização subcondral tardia e lentamente progressiva dessa vizinhança, outros autores mostram que também há aumento da mineralização nos segmentos teoricamente artrodesados. ${ }^{18}$ Inicialmente, mas não precocemente, há desmineralização dos segmentos fixados e adjacentes, tanto pela dor quanto pela diminuição das atividades. A persistência cíclica de cargas nessas facetas e a incapacidade de suportar o estresse oferecido nas vértebras vizinhas ao instrumental explicariam uma tendência das articulações facetarias desses níveis em mostrar alterações artríticas crônicas, degeneração histológica na cartilagem articular e, por conseguinte, instabilidade das já degeneradas vértebras. Hipotetiza-se que a desproporção entre o aumento do estresse externo e a redução da capacidade de carga dos níveis adjacentes pela redução da mineralização subcondral compensatória levaria a alterações degenerativas e, portanto, a fraturas nos níveis adjacentes; opinião compartilhada pelos autores.

Entretanto, essas alterações biomecânicas não foram acompanhadas de piora funcional desse grupo de pacientes, medida por meio da aplicação da Escala Analógica de Dor. Na comparação dos valores obtidos para a Escala Analógica de Dor e, indiretamente, por conseguinte, da liberdade funcional dos pacientes, houve melhora de quatro vezes entre a dor pré-operatória e a pós-operatória tardia $(\mathrm{p}<0,05)$, estatisticamente significativa. Em todos houve redução em pelo menos três pontos do grau de dor pré-operatória em relação ao pós-operatório tardio. Esse fato é mais evidente ainda naqueles pacientes sem ganho social (pacientes trabalhadores autônomos), que mostraram as melhores evoluções da dor no período. Isso mostra a clara influência que o meio exerce na dor e na capacidade funcional do indivíduo. No entanto, ao analisar-se a dor do paciente biomecanicamente e aceitando-se que os segmentos artrodesados não estão completamente fixos, a associação de micromovimento, espasmo da musculatura paravertebral doloroso, também poderia explicar a limitação funcional de alguns desses pacientes. Tal fato ainda necessita de maiores estudos.

\section{Conclusão}

A colocação de instrumentais curtos nas artrodeses toracolombares por via posterior não trouxe diferença entre os ângulos de deformidade sagital pós-operatória precoce e tardia, mostrando que o segmento mantém-se estável. Na deformidade sagital regional, parece não haver alteração cifótica significativa, mas justifica atenção maior, pela carga excessiva oferecida aos níveis adjacentes. Independentemente das alterações biome- 
cânicas, a artrodese pela via posterior é uma proposta alternativa de tratamento quando se discute a dor desses pacientes, reduzindo em pelo menos quatro vezes a dor no pós-operatório tardio. Fica a necessidade de abranger um número maior de pacientes com essa patologia, a fim de dispor dados mais fidedignos quanto à relação entre alterações biomecânicas e degenerativas tardias vertebrais no segmento toracolombar e o grau de liberdade funcional desses pacientes, respaldando o tratamento como uma alternativa viável no manejo de pacientes com fraturas toracolombares e neurologicamente intactos.

\section{Referências}

1. Grazier KL, Holbrook TL, Kelsey JL. The frequency of occurrence, impact, and cost of musculoskeletal conditions in the United States. Chicago: American Academy of Orthopedic Surgeons; 1984.

2. Pinto LG. Lesões traumáticas raquimedulares agudas: conceitos atuais. Rev Col Bras Cir. 1982;09(3):116-9.

3. Gertzbein SD. Scoliosis Research Society. Multicenter spine fracture study. Spine (Phila Pa 1976). 1992;17(5):528-40.

4. Markolf KL. Deformation of the thoracolumbar intervertebral joints in response to external loads: a biomechanical study using autopsy material. J Bone Joint Surg Am. 1972;54(3):511-33.

5. Oxland TR, Lin RM, Panjabi MM. Three-dimensional mechanical properties of the thoracolumbar junction. $\mathrm{J}$ Orthop Res. 1992;10(4):573-80.

6. Magerl F, Aebi M, Gertzbein SD, Harms J, Nazarian S. A comprehensive classification of thoracic and lumbar injuries. Eur Spine J. 1994;3(4):184-201.

7. McLain RF. The biomechanics of long versus short fixation for thoracolumbar spine fractures. Spine (Phila Pa 1976). 2006;31(11 Suppl):S70-9.

8. Anderson PA. Nonsurgical treatment of patients with thoracolumbar fractures. Instr Course Lect. 1995;44:57-65.

9. Cantor JB, Lebwohl NH, Garvey T, Eismont FJ. Nonoperative management of stable thoracolumbar burst fractures with early ambulation and bracing. Spine (Phila Pa 1976). 1993;18(8):971-6.
10. Kaneda K, Taneichi H, Abumi K, Hashimoto T, Satoh S, Fujiya M. Anterior decompression and stabilization with the Kaneda device for thoracolumbar burst fractures associated with neurological deficits. J Bone Joint Surg Am. 1997;79(1):69-83.

11. Siebenga J, Leferink VJ, Segers MJ, Elzinga MJ, Bakker FC, Haarman HJ, et al. Treatment of traumatic thoracolumbar spine fractures: a multicenter prospective randomized study of operative versus nonsurgical treatment. Spine (Phila Pa 1976). 2006;31(25):2881-90.

12. Vaccaro AR, Lim MR, Hurlbert RJ, Lehman RA Jr, Harrop $J$, Fisher DC, et al. Surgical decision making for unstable thoracolumbar spine injuries: results of a consensus panel review by the Spine Trauma Study Group. J Spinal Disord Tech. 2006;19(1):1-10.

13. Bracken MB, Freeman DH Jr, Hellenbrand K. Incidence of acute traumatic hospitalized spinal cord injury in the United States, 1970-1977. Am J Epidemiol. 1981;113(6):615-22.

14. Masini M. Tratamento das fraturas e luxações da coluna toracolombar por descompressão posterolateral e fixação posterior com retângulo e fios segmentares sublaminares associados a enxerto ósseo [dissertação]. São Paulo: Escola Paulista de Medicina; 2000.

15. Souza MF, Bastos BPR, Jallageas DN, Medeiros AAA. Perfil epidemiológico de 80 pacientes com traumatismo raquimedular, internados no Hospital do Pronto-Socorro Municipal de Belém, PA, no período de janeiro a setembro de 2002. J Bras Neurocirurg. 2002;13(3):92-8.

16. Zaninelli EM, Graells XSI, Néri OJ, Dau L. Avaliação epidemiológica das fraturas da coluna torácica e lombar de pacientes atendidos no Pronto-Socorro do Hospital do Trabalhador da UFPR de Curitiba - Paraná. Coluna/ Columna. 2005;4(1):11-5.

17. Campos MF, Ribeiro AT, Listik S, Pereira CAB, Sobrinho JA, Rapoport A. Epidemiologia do traumatismo da coluna vertebral. Rev Col Bras Cir. 2008;35(2):88-93.

18. Wagner S, Weckbach A, Müller-Gerbl M. The influence of posterior instrumentation on adjacent and transfixed facet joints in patients with thoracolumbar spinal injuries: a morphological in vivo study using computerized tomography osteoabsorptiometry. Spine (Phila Pa 1976). 200;30(7):E169-78.

\section{Endereço para correspondência}

Tiago de Paiva Cavalcante

Rua Professor Oséas Santos, nº 133, Edificio Camila, 102 - Amaralina -

Salvador - Bahia - Brasil.

E-mail: tiagoneuro@hotmail.com 